\title{
SOLUTIONS TO COOPERATIVE GAMES WITHOUT SIDE PAYMENTS
}

\author{
BY \\ BEZALEL PELEG
}

An extension of Von Neumann Morgenstern solution theory to cooperative games without side payments has been outlined in [1]. In this paper we revise some of the definitions given in [1] and prove that in the new theory every threeperson constant sum game is solvable (see [1, Theorem 1]). Other results that were formulated in [1] had already been proved in [2]. [1;2] are also necessary for a full understanding of the basic definitions of this paper.

1. Basic definitions. If $N$ is a set with $n$ members, we denote by $E^{N}$ the $n$-dimensional euclidean space the coordinates of whose points are indexed by the members of $N$. Subsets of $N$ will be denoted by $S$. If $x \in E^{N}$ and $i \in N, x^{i}$ will denote the coordinate of $x$ corresponding to $i ; x^{S}$ will denote the set $\left\{x^{i}: i \in S\right\}$. The superscript $N$ will be omitted, thus we write $x$ instead of $x^{N}$. We write $x^{s} \geqq y^{s}$ if $x^{i} \geqq y^{i}$ for all $i \in S$; similarly for $>$ and $=$. $\emptyset$ denotes the empty set.

Definition 1.1. An $n$-person characteristic function is a pair $(N, v)$ where $N$ is a set with $n$ members, and $v$ is a function that carries each $S \subset N$ into a set $v(S) \subset E^{N}$ so that

(1) $v(S)$ is closed,

(2) $v(S)$ is convex,

(3) $v(\varnothing)=E^{N}$,

(4) if $x \in v(S)$ and $x^{s} \geqq y^{s}$ then $y \in v(S)$.

Definition 1.2. An $n$-person game is a triad $(N, v, H)$, where $(N, v)$ is an $n$ person characteristic function and $H$ is a convex compact subset of $v(N)$.

We notice that this definition is not identical with that given in $[1 ; 2]$. In the first place $v$ is not assumed to be superadditive, i.e., the condition: $v\left(S_{1} \cup S_{2}\right)$ $\supset v\left(S_{1}\right) \cap v\left(S_{2}\right)$ for every pair of disjoint coalitions $S_{1}$ and $S_{2}$ is dropped. Secondly $H$ need not be a polyhedron.

2. Solutions. Let $G=(N, v, H)$ be an $n$-person game.

Definition 2.1. Let $x, y \in E^{N}, S \neq \varnothing$. $x$ dominates $y$ via $S$, written $x \succ_{s} y$, if $x \in v(S)$ and $x^{S}>y^{S}$.

Definition 2.2. $x$ dominates $y$, written $x \zeta_{y}$, if there is an $S$ such that $x \succ_{s} y$.

For $x \in E^{N}$ the following sets are defined: $\operatorname{dom}_{s} x=\left\{y: x \succ_{s} y\right\}$ and dom $x=\{y$ : $x \succ y\}$. Let $K \subset E^{N}$. We define $\operatorname{dom}_{S} K=\bigcup_{x \in K} \operatorname{dom}_{S} x$ and $\operatorname{dom} K=\bigcup_{x \in K} \operatorname{dom} x$.

Received by the editors June 28, 1961 and, in revised form, February 8, 1962. 
DEFINITION 2.3. $V$ is $K$-stable if $V=K-\operatorname{dom} V$.

Definition 2.4. The $K$-core is the set $K$-dom $K$.

We use the following abbreviation: P.S.O. - the proof, which is straightforward, will be omitted.

Proposition 2.5. Every K-stable set contains the K-core. P.S.O.

Proposition 2.6. If for each $x \in K \cap \operatorname{dom} K$ there is a $y \in K-\operatorname{dom} K$ such that $y \succ x$ then the K-core is the only K-stable set. P.S.O.

We denote: $v^{i}=\sup _{x \in v(\{i\})} x^{i}$.

Definition 2.7. $x$ is individually rational if $x^{i} \geqq v^{i}$ for all $i \in N$.

DeFInITION 2.8. $x$ is group rational if there is no $y \in H$ such that $y>x$.

We denote: $\bar{A}=\{x: x \in H, x$ is individually rational $\}$ and $A=\{x: x \in \bar{A}, x$ is group rational\}.

Proposition 2.9. $K$ is $A$-stable if and only if it is A-stable.

Proof. Let $K$ be $A$-stable. We show firstly that (1) $A-A \subset \operatorname{dom} K$. If $x \in$ $\bar{A}-A$ then there is a $y_{0} \in \bar{A}$ such that $y_{0}>x$. Define $f(y)=\min _{i \in N}\left(y^{i}-x^{i}\right)$. Since $f$ is continuous and $A$ is compact $f$ receives its maximum in $\bar{A}$ at a point $z$, which must be in $A$. By $1.2 z \in v(N) . f(z) \geqq f\left(y_{0}\right)$; therefore $z>x$. We have that $z \succ{ }_{N} x$ and if $w \succ z$ then $w \succ x$. If $z \in K$ then $x \in \operatorname{dom} K$. If $z \in \operatorname{dom} K$ then there is a $w_{0} \in K$ such that $w_{0} \succ z$ and therefore $w_{0} \succ x$, so $x \in \operatorname{dom} K$. From (1) it follows that $\bar{A}-\operatorname{dom} K=A-\operatorname{dom} K$ and therefore $K$ is $A$-stable. Now, let $K$ be $A$ stable. If $x \in \bar{A}-A$ we define $z$ as before and we see that $z \in A \subset K \cup \operatorname{dom} K$ implies that $x \in \operatorname{dom} K$. We conclude that (1) holds and therefore $K=A$-dom $K$ $=\bar{A}-\operatorname{dom} K$, i.e., $K$ is $\bar{A}$ stable.

Definition 2.10. A solution of $G$ is an $A$-stable set.

If $G$ has a solution we say that $G$ is solvable.

THEOREM 2.11. Every two person game has a unique solution, consisting of all of A. P.S.O.

Definition 2.12. $G$ is constant-sum if $H$ is contained in a plane

$$
\sum_{i \in N} x^{i}=e
$$

3. Three-person constant sum games.

I. Auxiliary lemmas. We use the following abbreviations: 3-P.C.G. three-person constant sum game, W. L. G. - without loss of generality.

Let $G=(N, v, H)$ be 3-P.C.G. We denote the members of $N$ by the first three positive integers and set $S_{i}=N-\{i\}$ for $i=1,2,3$. Let $x \in H$. We denote: $\sum_{i=1}^{3} x^{i}=e$ and $L=\left\{y: \sum_{i=1}^{3} y^{i}=e\right\}$. We have that $\bar{A}=\left\{x: x \in H, x^{i} \geqq v^{i}\right.$, $i=1,2,3\}=A$. So $A$ is a convex compact subset of $L$. Domination between 
points of $A$ is possible only via the $S_{i}$, i.e., if $x, y \in A$ and $x \succ_{s} y$ then $S$ is one of the $S_{i}$. For a subset $B$ of $L$ and $i \in N$ the following sets are defined: $B^{i}=B \cap v\left(S_{i}\right)$, $\dot{B}^{i}=B^{i} \cap \operatorname{dom}_{S_{i}} B^{i}$ and $b^{i}=B^{i}-\dot{B}^{i}$.

LEMMA I.1. If $B$ is convex then $B^{i}$ is convex.

Proof. $B^{i}=B \cap v\left(S_{i}\right)$ is convex. If $x_{1}, x_{2} \in B^{i}$ then there are $y_{1}, y_{2} \in B^{i}$ such that $y_{j} \succ_{s_{i}} x_{j}$ for $j=1,2$. If $0<t<1, x=t x_{1}+(1-t) x_{2}$ and $y=t y_{1}+(1-t) y_{2}$ then $x, y \in B^{i}$ and $y \succ_{s_{i}} x$, so $x \in \stackrel{B}{ }^{i}$.

We remark that $A^{i}$ is convex and compact, $\AA^{i}$ is convex and $a^{i}$ is compact.

Let $x \in L$ and $\varepsilon>0$. The set $\left\{y: y \in L, \sum_{i=1}^{3}\left(y^{i}-x^{i}\right)^{2}<\varepsilon^{2}\right\}$ is denoted by $S(x, \varepsilon), x$ is an interior point of a subset $B$ of $L$ if there is an $\varepsilon>0$ such that $S(x, \varepsilon) \subset B$.

LEMmA I.2. If $B \subset L$ is convex and $K=B^{i} \cap B^{j} \neq \varnothing, i \neq j$, then $K$ contains an interior point.

Proof. W.L.G. $i=1$ and $j=2$. We show firstly that $K \neq \varnothing$ implies that $B$ contains an interior point. If $B$ has no interior points then there are points $x_{1}$ and $x_{2}$ such that every $y \in B$ can be written as $y=t x_{1}+(1-t) x_{2},-\infty<t<\infty$. Let $x \in K . x=t_{0} x_{1}+\left(1-t_{0}\right) x_{2}$. There are $y_{l}=t_{l} x_{1}+\left(1-t_{l}\right) x_{2}, y_{l} \zeta_{s_{l}} x$ for $l=1,2$. We have $y_{1}^{2}>x^{2}$ and $y_{1}^{3}>x^{3}$, i.e., $t_{1} x_{1}^{2}+\left(1-t_{1}\right) x_{2}^{2}>t_{0} x_{1}^{2}+\left(1-t_{0}\right) x_{2}^{2}$ and $t_{1} x_{1}^{3}+\left(1-t_{1}\right) x_{2}^{3}>t_{0} x_{1}^{3}+\left(1-t_{0}\right) x_{2}^{3}$. So $\left(t_{1}-t_{0}\right)\left(x_{1}^{2}-x_{2}^{2}\right)>0$ and $\left(t_{1}-t_{0}\right)$ $\left(x_{1}^{3}-x_{2}^{3}\right)>0$. Therefore $\operatorname{sgn}\left(x_{1}^{2}-x_{2}^{2}\right)=\operatorname{sgn}\left(x_{1}^{3}-x_{2}^{3}\right)$. In the same way $y_{2} \succ_{s_{2}} x$ implies that $\operatorname{sgn}\left(x_{1}^{1}-x_{2}^{1}\right)=\operatorname{sgn}\left(x_{1}^{3}-x_{2}^{3}\right)$. So the three differences $x_{1}^{k}-x_{2}^{k}$ have the same sign, which is impossible since $\sum_{k=1}^{3} x_{1}^{k}=\sum_{k=1}^{3} x_{2}^{k}$. Now, let $z$ be an interior point of $B$ and $y \in K$. For small positive $t$ the points $t z+(1-t) y$ are interior points of $K$.

Lemma I.3. If $x \in O=\bigcap_{i=1}^{3} \AA^{i}$ then $x$ is an interior point of $O$.

Proof. There are $y_{j} \succ_{s_{j}} x$ for $j=1,2,3$. We have: $y_{1}^{2}>x^{2}, y_{1}^{3}>x^{3}, y_{2}^{1}>x^{1}$, $y_{2}^{3}>x^{3}, y_{3}^{1}>x^{1}$ and $y_{3}^{2}>x^{2}$. There exist $0<t_{k}<1$ such that $z_{k}=t_{k} y_{1}+\left(1-t_{k}\right) y_{k}$ satisfy $z_{k}^{1}=x^{1}, k=2,3$. Since $z_{2}^{3}>x^{3}$ and $z_{3}^{3}<x^{3}$ there is a $0<t_{1}<1$ such that $x=t_{1} z_{2}+\left(1-t_{1}\right) z_{3}$. So $x$ is an interior point of the convex hull of $\left\{y_{1}, y_{2}\right.$, $\left.y_{3}\right\}$ and therefore of $A$. But if $x \in O$ is an interior point of $A$ then $x$ is also an interior point of $O$.

LEMMA I.4. If $B \subset L$ is convex, $x_{1}, x_{2} \in B, x_{1}^{k}=x_{2}^{k}, x_{1}^{i}<x_{2}^{i}, x_{1}^{j}>x_{2}^{j}$ and $y$ satisfies $y^{i}=x_{2}^{i}, y^{j}=x_{1}^{j}$ and $y^{k}=x_{1}^{i}+x_{1}^{k}-x_{2}^{i}$ then: $y \notin B$ if and only if $B \cap\left\{z: z^{S_{k}} \geqq y^{S_{k}}\right\}=\varnothing$. P.S.O.

LEMmA I.5. If $x \in \AA^{i}$ then there is $a y \in a^{i}$ such that $y \succ_{s_{i}} x$ and for every $\varepsilon>0, S(y, \varepsilon) \cap \AA^{i} \neq \varnothing$.

Proof. Define $f(z)=\min _{j \in S_{i}}\left(z^{j}-x^{j}\right) . f$ receives its maximum in $A^{i}$ at a point 
$y$ which must be in $a^{i}$. Since $x \in \AA^{i} f(y)>0$ and therefore $y \succ_{s_{1}} x$. If $0 \leqq t<1$ then $t y+(1-t) x$ is in $\AA^{i}$, therefore for every $\varepsilon>0 S(y, \varepsilon) \cap \AA^{i} \neq \varnothing$.

If $x, y \in L$ then the set $\{z: z=t x+(1-t) y, 0 \leqq t \leqq 1\}$ is denoted by $[x y]$ and is called an interval. $x$ and $y$ are called the ends of $[x y] \cdot(x y]=[x y]-\{x\}$ $\cdot[x y)=[x y]-\{y\} \cdot(x y)=[x y]-(\{x\} \cup\{y\})$. For $i=1,2,3$ the following sets are defined: $D^{i}=A^{j} \cap A^{k}$, where $S_{i}=\{j, k\}$, and $F^{i}=\left\{x: x \in D^{i}, x^{i} \geqq y^{i}\right.$ for every $\left.y \in D^{i}\right\} \cdot D^{i}$ is convex and compact. $F^{i}$ is an interval.

LEMMA I.6. Let $S_{k}=\{i, j\}$. If $x^{i}$ receives its maximum in $F^{k}$ at a point $\alpha$, then: $\alpha \notin a^{j}$ if and only if $\stackrel{\circ}{ }^{j} \supset A^{i}$.

Proof. W.L.G. $i=2$ and $j=3$. If $A^{3} \supset A^{2}$ then $\alpha \in F^{1} \subset D^{1} \subset A^{2} \subset A^{3}$. If $\alpha \notin a^{3}$ then there is an $x \in A^{3}$ such that $x \succ_{S_{3}} \alpha$. There is an $\varepsilon>0$ such that $U$ $=S(\alpha, \varepsilon) \cap A \subset \dot{A}^{3}$. Now we show that if $y \in A^{2}$ then $y^{1} \leqq \alpha^{1}$. If there is a $z \in A^{2}$ such that $z^{1}>\alpha^{1}$ then for a small positive $t u=t z+(1-t) \alpha$ satisfies $u^{1}>\alpha^{1}$ and $u \in U$. So we have $u \in D^{1}$ and $u^{1}>\alpha^{1}$ which is impossible. Next we show that $y \in A^{2}$ implies that $y^{2} \leqq \alpha^{2}$. Suppose that there is a $z \in A^{2}$ such that $z^{2}>\alpha^{2}$. If $z^{1}=\alpha^{1}$ then for a small positive $t u=t z+(1-t) \alpha$ satisfies $u \in U, u^{1}=\alpha^{1}$ and $u^{2}>\alpha^{2}$. So we have that $u \in F^{1}$ and $u^{2}>\alpha^{2}$ which is impossible. If $z^{1}<\alpha^{1}$ then there is a $0<t<1$ such that $w=t z+(1-t) x$ satisfies $w^{1}=\alpha^{1}$ and $w^{2}>\alpha^{2}$ $\cdot \alpha^{S_{2}} \geqq w^{S_{2}}$ therefore $w \in A^{2}$, but this is impossible as we have already shown. We have shown that every $y \in A^{2}$ satisfies $y^{S_{3}} \leqq \alpha^{S_{3}}$. Since $\alpha \in \operatorname{dom}_{S_{3}} x$ we have $A^{2} \subset A \cap \operatorname{dom}_{S_{3}} x \subset \AA^{3}$.

The sets $\left\{A^{1}, A^{2}\right\},\left\{A^{2}, A^{3}\right\}$ and $\left\{A^{3}, A^{1}\right\}$ will be called pairs.

DeFINITION I.7. The pair $\left\{A^{i}, A^{j}\right\}$ intersects maximally if:

(1) $\AA^{i} \cap \stackrel{\circ}{ }^{j} \neq \varnothing$.

(2) $a^{i} \cap a^{j} \neq \varnothing$.

The number of pairs that intersect maximally will be denoted by $m(G)$.

Lemma I.8. Let $i \neq j$ and $\AA^{i} \cap \stackrel{\circ}{j}^{j} \neq \varnothing . \AA^{i} \neq A^{j}$ and $\stackrel{\circ}{A}^{j} \neq A^{i}$ if and only if $\left\{A^{i}, A^{j}\right\}$ intersects maximally.

Proof. W.L.G. $i=2$ and $j=3$. If $\AA^{3} \supset A^{2}$ or $\AA^{2} \supset A^{3}$ then $a^{2} \cap a^{3}=\varnothing$ and therefore $\left\{A^{2}, A^{3}\right\}$ does not intersect maximally. Now suppose that $\AA^{2} \ngtr A^{3}$ and $\AA^{3} \ngtr A^{2}$. Let $x^{2}$ and $x^{3}$ receive their maxima in $F^{1}$ at the points $\alpha$ and $\beta$ respectively. By I.6: $A^{2} \notin \AA^{3}$ implies that $\alpha \in a^{3}$ and $\AA^{2} \neq A^{3}$ implies that $\beta \in a^{2}$. We have $\left({ }^{1}\right)$ that $F^{1} \subset a^{2} \cup a^{3}$ and $F^{1} \cap a^{k} \neq \varnothing, k=2,3$. Since $F^{1}$ is connected and $F^{1} \cap a^{3}$ and $F^{1} \cap a^{2}$ are closed we must have $\left(F^{1} \cap a^{2}\right) \cap\left(F^{1} \cap a^{3}\right)=F^{1}$ $\cap a^{2} \cap a^{3} \neq \varnothing$.

From the proof of I.8 we can conclude that: (I.9) if $\left\{A^{i}, A^{j}\right\}$ intersects maximally then $F^{k} \cap a^{i} \cap a^{j} \neq \emptyset$ where $\{k\}=N-\{i, j\}$.

(1) Otherwise there is $x \in F^{1} \cap \stackrel{\circ}{A}^{2} \cap \stackrel{\circ}{A}^{3}$. Let $z \succ s_{2} x$. For small $t>0, u=t z+(1-t) x$ satisfy $u^{1}>x^{1}$ and $u \in D^{1}$, which is impossible. 
LEMMA I.10. If $i \neq j, x, y \in a^{i}, x \neq y$ and $x^{j}=y^{j}$ then every $z \in A^{i}$ satisfies $z^{j} \leqq x^{j}$ and $\left\{u: u \in A^{i}, u^{j}=x^{j}\right\} \subset a^{i}$. P.S.O.

LEMMA I.11. Let $S_{k}=\{i, j\}$. If $\left\{A^{i}, A^{j}\right\}$ intersects maximally and $x^{i}$ and $x^{j}$ take their maxima in $F^{k}$ at the points $\alpha$ and $\beta$ respectively then $F^{k}=[\alpha \beta]$ and one of the following possibilities holds:
(a) $\alpha=\beta$,
$\alpha \in a^{i} \cap a^{j}$
(b) $\alpha \neq \beta$,
$[\alpha \beta] \subset a^{i} \cap a^{j}$,
(c) $\alpha \neq \beta$,
$(\alpha \beta] \subset a^{i} \cap \AA^{j}$,
(c) $\alpha \neq \beta$,
$[\alpha \beta) \subset a^{j} \cap \stackrel{\circ}{A}^{i}$,
$\alpha \in a^{i} \cap a^{j}$, $\beta \in a^{i} \cap a^{j}$.

Proof. W.L.G. $i=2$ and $j=3$. We saw in the proof of I.8 that $\alpha \in F^{1} \cap a^{3}$ and $\beta \in F^{1} \cap a^{2}$. If $\alpha=\beta$ then (a) holds. If $\alpha \neq \beta$ we have the following possibilities for the relative positions of $a^{3}$ and $F^{1}$ :

(1) There is no $x \neq \alpha$ in $a^{3} \cap F^{1}$, i.e., $(\alpha \beta] \subset \AA^{3}$.

(2) There is an $x \neq \alpha$ in $a^{3} \cap F^{1}$, and, therefore, by I.10, $F^{1} \subset a^{3}$.And similarly for $a^{2}$ and $F^{1}$ :

(3) There is no $y \neq \beta$ in $a^{2} \cap F^{1}$, i.e., $[\alpha \beta) \subset \AA^{2}$.

(4) There is a $y \neq \beta$ in $a^{2} \cap F^{1}$ and therefore $F^{1} \subset a^{2}$.

Since $F^{1} \subset a^{2} \cup a^{3}$ (1) and (3) cannot hold together. If (2) and (4) hold together then we have (b). If (1) and (4) hold together, then we have ( $\left.c_{j}\right)$. If (2) and (3) hold together then we have $\left(\mathrm{c}_{i}\right)$. We say that $F^{k}$ has $a$-shape if (a) holds; similarly for (b), $\left(\mathrm{c}_{i}\right)$ and $\left(\mathrm{c}_{j}\right)$.

For $x \in A$ the following sets are defined: $Q_{i}(x)=\left\{y: y \in A, y^{S_{t}} \geqq x^{S_{t}}\right\}, T_{i}(x)$ $=\left\{y: y \in A, y^{i}<x^{i}\right\}$ and $R_{i}(x)=A-T_{i}(x)$. We remark that:

(I.12) $\operatorname{dom} Q_{i}(x) \cap R_{i}(x)=\operatorname{dom}_{s_{i}} Q_{i}(x) \cap R_{i}(x)$,

(I.13) $x \notin \AA^{i}$ if and only if $x \in Q_{i}(x)-\operatorname{dom} Q_{i}(x)$,

(I.14) $x \notin \AA^{i}$ if and only if $Q_{i}(x) \cap \AA^{i}=\emptyset$.

LEMMA I.15. If $x \in A-A^{\circ}, y \neq x, y \in A^{i} \cap Q_{i}(x)$ then there is $a j \in S_{i}$ such that every $z \in A^{i}$ satisfies $z^{j} \leqq x^{j}$. P.S.O.

LEMMA I.16. Let $S_{k}=\{i, j\}$. We denote the ends of $F^{k}$ by $\alpha$ and $\beta$ such that $\alpha^{i} \geqq \beta^{i}$. If $\gamma \in A$ satisfies $\gamma^{k}=\alpha^{k}$ and $\gamma^{i}<\beta^{i}$ then $Q_{i}(\gamma) \cap A^{i}=\emptyset$. P.S.O.

LEMMA I.17. Let $S_{k}=\{i, j\}$. If $\gamma \in F^{k} \cap a^{i} \cap a^{j}$ and $x \in R_{k}(\gamma)-F^{k}$ then $\operatorname{dom} x \cap Q_{k}(\gamma)=\emptyset$.

Proof. W.L.G. $i=2$ and $j=3$. We denote the ends of $F^{1}$ by $\alpha$ and $\beta$ such that $\alpha^{2} \geqq \beta^{2}$. Let $x \in R_{1}(\gamma)-F^{1}$ and $y \in Q_{1}(\gamma) \cdot x^{2}+x^{3} \leqq \gamma^{2}+\gamma^{3} \leqq y^{2}+y^{3}$ so $x \succ_{s_{1}} y$ is impossible. If $x^{1}>\gamma^{1}$ and $x \succ_{s_{2}} y$ or $x \succ_{s_{3}} y$ then $x \succ_{s_{2}} y$ or $x \succ_{s_{3}} \gamma$ respectively, which is impossible. If $x^{1}=\gamma^{1}$ then either $x^{2}<\beta^{2}$ or $x^{3}<\alpha^{3}$. If $x^{3}<\alpha^{3}$ then, by I.16, $x \notin A^{3}$. Since $y^{3} \geqq \gamma^{3} \geqq \alpha^{3}>x^{3}$, if $x \succ y$ then $x \succ_{s_{3}} y$, but this is impossible. Similarly if $x^{2}<\beta^{2}$ then $x \succ y$ is impossible. 
LEMmA I.18. If $a B \subset A$ is convex and compact then $(N, v, B)$ is 3-P.C.G., $B^{i}=A^{i} \cap B$ and $\stackrel{\circ}{B}^{i} \subset \stackrel{\circ}{ }^{i} \cap$ B. P.S.O.

If $B \subset A$ is convex and compact we say that $B$ is solvable or that $B$ has $a$ solution if $(N, v, B)$ is solvable. We also write $m(B)$ instead of $m((N, v, B))$.

LEMMA 1.19. If $x \in D^{k}$ and $l \in S_{k}$ then $\stackrel{\circ}{k}_{k}^{l}(x)=\AA^{l} \cap \cap R_{k}(x)$.

Proof. If $y \in \AA^{l} \cap R_{k}(x)$ then $y^{k} \geqq x^{k}$ and there is a $z \in A^{l}$ such that $z \zeta_{s_{1}} y$. Since $z^{k}>y^{k} z \in R_{k}(x)$. So we have $z \in R_{k}(x) \cap A^{l}=R_{k}^{l}(x)$ and therefore $y \in \check{R}_{k}^{l}(x)$. We have shown that $\dot{R}_{k}^{l}(x) \supset \AA^{l} \cap R_{k}(x)$. By I.18 $\dot{R}_{k}^{l}(x) \subset \AA^{l} \cap R_{k}(x)$, so $\stackrel{\circ}{k}_{k}^{l}(x)=\stackrel{\circ}{A}^{l} \cap R_{k}(x)$.

LEMma I.20. If $x \in D^{k}-\AA^{k}, l \in S_{k}$ and $\left\{A^{k}, A^{l}\right\}$ does not intersect maximally, then $\left\{R_{k}^{1}(x), R_{k}^{k}(x)\right\}$ does not intersect maximally.

Proof. Since $\left\{A^{k}, A^{i}\right\}$ does not intersect maximally, by I.8 at least one of the the following possibilities holds: $\AA^{k} \cap \AA^{l}=\varnothing, \AA^{l} \supset A^{k}$ or $\AA^{k} \supset A^{l}$. If $\AA^{k} \cap \AA^{l}=\varnothing$ then $\stackrel{\circ}{k}_{k}^{k}(x) \cap \check{R}_{k}^{l}(x) \subset A^{\circ} \cap \AA^{l} \cap R_{k}(x)=\emptyset$. If $A^{i} \supset A^{k}$ then $\dot{R}_{k}^{l}(x)=\AA^{l} \cap R_{k}(x)$ $\supset A^{k} \cap R_{k}(x)=R_{k}^{k}(x) . A^{k} \supset A^{l}$ is impossible since $x \in A^{l}-A^{k}$.

Definition I.21. Let $B_{1}, \cdots, B_{l}$ be convex compact subsets of $A . B_{1}, \cdots, B_{l}$ are called independent if there exist solutions $V_{1}, \cdots, V_{l}, V_{i}$ solution of $B_{i}$ respectively, such that $\operatorname{dom} V_{k} \cap\left(\bigcup_{j=1}^{l} V_{j}\right)=\emptyset$ for $k=1, \cdots, l$.

Lemma I.22. If $B_{1}, \cdots, B l$ are independent then there exist solutions $V_{1}, \cdots, V_{l}$, $V_{i}$ solution of $B_{i}$ for $i=1, \cdots, l$, such that $\bigcup_{j=1}^{l} V_{j}$ is $\bigcup_{j=1}^{l} B_{j}$-stable. P.S.O.

In the following three subsections we shall prove:

THEOREM. Every 3-P.C.G. G is solvable.

The proof will be by induction on $m(G)$.

II. First part: $m(G)=0$. In this subsection we show that every 3-P.C.G. $G$ for which $m(G)=0$ is solvable. We also prove some additional auxiliary lemmas:

Lemma II.1. Let $G$ be 3-P.C.G. If $\AA^{1} \cap \AA^{2}=A^{\circ} \cap \AA^{3}=\AA^{3} \cap \AA^{1}=\emptyset$ then the $A$-core is the solution of $G$.

Proof. Denote $C=A-\operatorname{dom} A$. If $x \in A-C$ then there is a $y \in A$ that dominates it. There is an $i$ such that $y \zeta_{s_{i}} x$, i.e., $x \in A^{i}$. By I.5 there is a $z \in a^{i}$ such that $z \zeta_{s_{1}} x$. If $z \notin C$ then $z \in \AA^{l}$ where $l \neq i$. There is an $\varepsilon>0$ such that $S(z, \varepsilon) \cap A$ $\subset \AA^{i}$. But $S(z, \varepsilon) \cap A^{\circ} \neq \varnothing$; therefore $\AA^{i} \cap \AA^{l} \neq \varnothing$ which is impossible. We have shown that for every $x \in A-C$ there is a $z \in C$ such that $z \zeta x$. By $2.6 C$ is the only $A$-stable set.

ILEMMA II.2. Let $G$ be 3-P.C.G. If $m(G)=0$ then the A-core is the solution of $G$.

Proof. If $\dot{A}^{1} \cap A^{2}=A^{2} \cap \AA^{3}=A^{3} \cap \dot{A}^{1}=\emptyset$ then by II.1 the $A$-core is the solution of $G$. If it is not the case then, W.L.G., we assume that $\AA^{2} \cap \AA^{3} \neq \varnothing$. Since $\left\{A^{2}, A^{3}\right\}$ does not intersect maximally we have that either $\AA^{3} \supset A^{2}$ or $\AA^{2} \supset A^{3}$. 
W.L.G. we suppose that $\AA^{3} \supset A^{2}$. There are three possibilities for the relative position of $A^{1}$ and $A^{3}$ : (a) $\AA^{1} \cap \AA^{3}=\varnothing$, (b) $\AA^{3} \supset A^{1}$ or (c) $\AA^{1} \supset A^{3}$. In each case we show that $C$, the $A$-core, is the solution of $G$. (a) $A^{1} \cap \AA^{3}=\varnothing$. If $x \in A-C$ then there is a $y \in A$ such that $y \succ_{s_{i}} x$ for some $i$. Since $\dot{A}^{3} \supset A^{2}$ we may assume that $i \in S_{2}$. There is a $z \in a^{i}$ such that $z \zeta_{s_{i}} x$. If $i=1$ then, since $\AA^{1} \cap \AA^{j}=\varnothing$ for $j=2,3, z \in C$. If $i=3$ then, since $a^{3} \cap \AA^{2}=\varnothing$ and $\AA^{1} \cap \AA^{3}=\varnothing, z \in C$. By $2.6 C$ is the solution of $G$. (b) $\AA^{3} \supset A^{1}$. If $x \in A-C$ then there is a $y \in A$ such that $y \succ_{s_{3}} x$. So there is a $z \in a^{3}$ such that $z \succ x$. Since $a^{3} \cap\left(\AA^{1} \cup \AA^{2}\right)=\varnothing, z \in C$. So $C$ is the solution of $G$. (c) $\AA^{1} \supset A^{3}$. The proof in this case parallels that in case (b).

Let $G=(N, v, H)$ be 3-P.C.G.

Lemma II.3. If $\xi \in D^{k}-\AA^{k}, \eta \in D^{k} \cap Q_{k}(\xi)$ and $U$ is a solution of $Q_{k}(\eta)$ then $V=U \cup[\xi \eta]$ is a solution of $Q_{k}(\xi)$.

Proof. W.L.G. $k=1$. $\eta^{1} \leqq \xi^{1}, \eta^{2} \geqq \xi^{2}$ and $\eta^{3} \geqq \xi^{3}$; therefore $\left(\operatorname{dom}_{S_{3}}[\xi \eta]\right.$ $\left.\cup \operatorname{dom}_{S_{2}}[\xi \eta]\right) \cap[\xi \eta]=\varnothing$. $\xi \cdots \AA^{1}$; therefore $Q_{1}(\xi) \cap \AA^{1}=\varnothing$. So $\operatorname{dom}_{s_{1}}[\xi \eta]$ $\cap[\xi \eta]=\varnothing$. Summing we have (1) dom $[\xi \eta] \cap[\xi \eta]=\varnothing$. Now we show (2) $Q_{1}(\xi)$ $-[\xi \eta]-Q_{1}(\eta) \subset \operatorname{dom}[\xi \eta]$. Let $x \in Q_{1}(\xi)-[\xi \eta]-Q_{1}(\eta)$. If $x^{1} \geqq \eta^{1}$ then there is a $y \in[\xi \eta]$ such that $y^{1}=x^{1} . y \neq x$ so we may assume that $y^{2}>x^{2}$. Under this assumption we can find a $z \in[\xi \eta]$ with $z^{S_{3}}>x^{S_{3}}$, so $z \zeta x$. If $x^{1}<\eta^{1}$ then, since $x \notin Q_{1}(\eta)$, either $x^{2}<\eta^{2}$ or $x^{3}<\eta^{3}$ and therefore $x \in \operatorname{dom}_{s_{3}} \eta \cup \operatorname{dom}_{s_{2}} \eta$. We now prove (3) dom $U \cap[\xi \eta]=\varnothing$ and $\operatorname{dom}[\xi \eta] \cap U=\varnothing$. Let $x \in[\xi \eta]$ and $y \in U$. $y^{1} \leqq x^{1}, y^{2} \geqq x^{2}$ and $y^{3} \geqq x^{3}$ therefore $x \succ y$ is impossible and if $y \succ x$ then $y \succ_{s_{1}} x$, but, since $x \notin \AA^{1}$, this is also impossible. Combining (1), (2) and (3) it follows that $V=U \cup[\xi \eta]$ solves $Q_{1}(\xi)$.

Lemma II.4. If $\xi \in D^{k}-\AA^{k}$ then $Q_{k}(\xi)$ is solvable and if $V$ solves it then $\operatorname{dom} V \supset T_{k}(\xi)-V$.

Proof. W.L.G. $k=1$. Denote $J=Q_{1}(\xi) \cap D^{1}$. Let $\eta$ be a point where $x^{1}$ receives its minimum in $J$. We show that $\grave{Q}_{1}^{i}(\eta) \cap \grave{Q}_{1}^{j}(\eta)=\varnothing$ for all $i \neq j$. First, since $\xi \notin \stackrel{\circ}{A}^{1}, \AA^{1} \cap Q_{1}(\xi)=\varnothing$. So we have $\grave{Q}_{1}^{1}(\eta) \subset \AA^{1} \cap Q_{1}(\eta) \subset \AA^{1} \cap Q_{1}(\xi)=\emptyset$. Next, since $x^{1}$ receives its minimum in $J$ at $\eta Q_{1}(\eta) \cap D^{1}=\{\eta\} . \eta \notin \AA^{1}$ therefore $\eta \in Q_{1}(\eta)$-core. We have $\grave{Q}_{1}^{2}(\eta) \cap \dot{Q}_{1}^{3}(\eta) \subset Q_{1}^{2}(\eta) \cap Q_{1}^{3}(\eta)=D^{1} \cap Q_{1}(\eta)=\{\eta\}$, so $\grave{Q}_{1}^{2}(\eta) \cap \grave{Q}_{1}^{3}(\eta)=\varnothing$. By II. $1 Q_{1}(\eta)$ is solvable. Let $U$ be a solution of $Q_{1}(\eta)$; by II.3 $U \cup[\xi \eta]$ solves $Q_{1}(\xi)$. Now let $V$ be a solution of $Q_{1}(\xi)$. $\xi \in Q_{1}(\xi)$-core so $\xi \in V . \operatorname{dom} \xi \supset T_{1}(\xi)-Q_{1}(\xi)$ therefore $\operatorname{dom} V \supset\left(Q_{1}(\xi)-V\right) \cup\left(T_{1}(\xi)-Q_{1}(\xi)\right)$ $=T_{1}(\xi)-V$.

LEMmA II.5. Let $S_{k}=\{i, j\}$. If $F^{k}$ has $c_{j}$-shape and $\mu \in F^{k} \cap A^{k}$ then there is a solution $V$ of $Q_{i}(\mu)$ such that $V \cap F^{k}=\{\mu\}$.

Proof. W.L.G. $i=2$ and $j=3$. We denote the ends of $F^{1}$ by $\alpha$ and $\beta$ such that $\alpha^{2}>\beta^{2}$. Since $F^{1}$ has $c_{3}$-shape we have (1) $\AA^{2} \cap R_{1}(\alpha)=\emptyset$ and $(2)[\beta \alpha) \subset \AA^{3}$. From (1) it follows that (3) $\grave{Q}_{2}^{2}(\mu)=\varnothing$. We show (4) $[\beta \mu) \subset \grave{Q}_{2}^{3}(\mu)$. Let $x \in[\beta \mu)$. 
$x^{1}=\mu^{1}, x^{2}<\mu^{2}$ and $x^{3}>\mu^{3}$. By (2) $x \in \AA^{3}$, therefore there is $a y \in A^{3}$ such that $y \succ_{s_{3}} x$. For small $t>0 \quad z=t y+(1-t) x$ satisfy $z^{1}>x^{1}, z^{2}>x^{2}$ and $z^{3}>\mu^{3}$, so $z \in Q_{2}^{3}(\mu)$ and $x \in \grave{Q}_{2}^{3}(\mu)$. For the relative position of $Q_{2}^{1}(\mu)$ and $Q_{2}^{3}(\mu)$ we have the following possibilities: (a) $\grave{Q}_{2}^{1}(\mu) \cap \grave{Q}_{2}^{3}(\mu)=\varnothing$ or (b) $\grave{Q}_{2}^{1}(\mu) \cap \grave{Q}_{2}^{3}(\mu) \neq \varnothing$. If (a) holds then by (3) $\dot{Q}_{2}^{i}(\mu) \cap \grave{Q}_{2}^{j}(\mu)=\varnothing$ for all $i \neq j$. By II.1 $Q_{2}(\mu)-\operatorname{dom} Q_{2}(\mu)$ is a solution of $Q_{2}(\mu)$ and since $\mu \in Q_{2}(\mu)$-core, $\left(Q_{2}(\mu)-\operatorname{dom} Q_{2}(\mu)\right) \cap F^{1}=\{\mu\}$. If (b) then by I.2 there is an interior point $\zeta$ of $\dot{Q}_{2}^{1}(\mu) \cap \grave{Q}_{2}^{3}(\mu) . \zeta^{1}>\mu^{1}, \zeta^{2}<\mu^{2}$ and $\zeta^{3}>\mu^{3} . \zeta \in D^{2}-A^{2}$, therefore by II.4 $Q_{2}(\zeta)$ is solvable. If $U$ is a solution of $Q_{2}(\zeta)$ then, by II.3, $[\zeta \mu] \cup U$ solves $Q_{2}(\mu)$. Since $([\zeta \mu] \cup U) \cap F^{1}=\{\mu\}$ this completes the proof.

Definition II.6. The pair $\left\{A^{i}, A^{j}\right\}$ satisfies condition $M$ if:

(1) $\left\{A^{i}, A^{j}\right\}$ intersects maximally,

(2) $F^{k} \cap a^{i} \cap a^{j} \& \AA^{k}$ where $\{k\}=N-\{i, j\}$.

We now formulate the induction hypothesis:

II.7. every 3-P.C.G. G for which $m(G) \leqq l-1$ is solvable. Let $G$ be 3-P.C.G. for which $m(G)=l$. We have to prove that $G$ is solvable. We distinguish between the following possibilities:

II.8. there is at least one pair that satisfies condition $M$.

II.9. there is no pair that satisfies condition $M$.

III. Second part: case II.8. W.L.G. $\left\{A^{2}, A^{3}\right\}$ satisfies condition $M$. The ends of $F^{1}$ will be denoted by $\alpha$ and $\beta$ such that $\alpha^{2} \geqq \beta^{2} . F^{1} \cap a^{2} \cap a^{3} \notin \AA^{1}$ therefore at least one of the ends is in $a^{2} \cap a^{3}-\AA^{1}$. We shall prove that $G$ is solvable when: (III.1) $\alpha \in a^{2} \cap a^{3}-\AA^{1}$. The proof when $\beta \in a^{2} \cap a^{3}-\AA^{1}$ is similar to that in case (III.1). We shall distinguish three cases according to the three possible shapes of $F^{1}$ in case (III.1).

III.a. $F^{1}$ has a-shape. By (III.1) and II.4 $Q_{1}(\alpha)$ is solvable and if $V$ solves it then (1) $\operatorname{dom} V \supset T_{1}(\alpha)-V$. Since $\alpha \in F^{1} \AA^{2} \cap \AA^{3} \cap R_{1}(\alpha)=\varnothing$. By I.19 $\AA_{1}^{2}(\alpha)$ $\cap \stackrel{\circ}{R}_{1}^{3}(\alpha)=\varnothing$, so $\left\{R_{1}^{2}(\alpha), R_{1}^{3}(\alpha)\right\}$ does not intersect maximally. From I.20 it follows now that $m\left(R_{1}(\alpha)\right) \leqq l-1$. By II.7 $R_{1}(\alpha)$ is solvable. If $Q_{1}(\alpha)$ and $R_{1}(\alpha)$ are independent then from I.22 and (1) it follows that $A$ has a solution. If $Q_{1}(\alpha)$ and $R_{1}(\alpha)$ are not independent then if $V$ solves $Q_{1}(\alpha)$ and $W$ solves $R_{1}(\alpha)$ either (2) $\operatorname{dom} V \cap W \neq \varnothing$ or (3) dom $W \cap V \neq \varnothing$. From III.a and I.17 it follows that (4) $\operatorname{dom} R_{1}(\alpha) \cap Q_{1}(\alpha)=\varnothing$. By (4) we have that (3) is impossible. By (III.1) $\alpha \in A$ - $\operatorname{dom} A$ therefore (5) $\alpha \in W \cap V$. From (2), (5) and I.12 it follows that there is a $z \neq \alpha$ in $V \cap A^{1}$. By I.15 and due to III.a, we may assume that every $y \in A^{1}$ satisfies $y^{2} \leqq \alpha^{2}$. Let $\zeta$ be a point where $x^{3}$ receives its maximum $\left({ }^{2}\right)$ in $V \cap A^{1}$. If $u \in V \cap A^{1}$ then $\zeta^{2}=u^{2}$ and $\zeta^{3} \geqq u^{3}$ and therefore (6) $\operatorname{dom}_{s_{1}} \zeta \supset \operatorname{dom}_{s_{1}} u$. $\alpha \in D^{3}-\AA^{3}$ therefore, by II.4, $Q_{3}(\alpha)$ is solvable. If $U$ solves $Q_{3}(\alpha)$ then by (4) we have that (7) $\operatorname{dom} U \cap V=\varnothing$. We remark that (8) $Q_{3}(\alpha) \cap A^{3} \subset\left\{x: x^{2}=\alpha^{2}\right\}$. Let $x^{1}$ receive its maximum in $U \cap A^{3}$ at the point $\eta$. We define: $v=\left(\eta^{1}, e-\eta^{1}\right.$ $\left.-\zeta^{3}, \zeta^{3}\right)$. By I.12 and (6) we have that (9) $R_{1}(\alpha)-\operatorname{dom} V=R_{1}(\alpha)$

(2) Observe that a solution of a compact set is compact. see [3, Theorem 3]. 
$-\operatorname{dom}_{s_{1}} \zeta=Q_{3}(\alpha) \cup\left\{x: x \in R_{1}(\alpha), x^{3} \geqq \zeta^{3}\right\}$. By (8) we have that (10) $Q_{2}(\alpha)$ $-\operatorname{dom} U=Q_{2}(\alpha)-\operatorname{dom}_{s_{3}} \eta=\left\{x: x \in Q_{2}(\alpha), x^{1} \geqq \eta^{1}\right\}$. Combining (1), (9) and (10) we have (11) $A-\operatorname{dom}(U \cup V)=\left\{x: x \in A, x^{S_{2}} \geqq v^{s_{2}}\right\}$. If $v \notin A$ then from I.4, (11), (9) and (7) it follows that $U \cup V$ solves $A$. Suppose now that $v \in A$. We define $v_{1}=\left(\alpha^{1}, e-\alpha^{1}-\zeta^{3}, \zeta^{3}\right)$. By I.16 we have that (12) $\varnothing=A^{2} \cap Q_{2}$ $\left(v_{1}\right) \supset A^{2} \cap Q_{2}(v) . Q_{1}(\alpha) \cup Q_{3}(\alpha) \subset R_{2}(v)$ therefore by (12) and I.12 we have (13) $\operatorname{dom} Q_{2}(v) \cap\left(Q_{1}(\alpha) \cup Q_{3}(\alpha)\right)=\varnothing$. $v \in D^{2}-A^{2}$ therefore $Q_{2}(v)$ is solvable. If $U_{1}$ solves $Q_{2}(v)$ then by (11) and (13) $V \cup U \cup U_{1}$ is a solution of $A$.

III.b. $F^{1}$ has $b$-shape. Due to III.b, we have that $R_{1}(\alpha) \cap\left(\AA^{2} \cup \AA^{3}\right)=\emptyset$ and therefore (1) $\stackrel{\circ}{1}_{1}^{2}(\alpha)=\stackrel{\circ}{R}_{1}^{3}(\alpha)=\emptyset$. From (1) it follows that (2) $R_{1}^{\circ}(\alpha) \cap \dot{R}_{1}^{j}(\alpha)=\varnothing$ for all $i \neq j$. If $x \in R_{1}(\alpha)$ then $x^{2}+x^{3} \leqq \alpha^{2}+\alpha^{3}$ therefore (3) $[\alpha \beta] \cap \operatorname{dom}_{s_{1}} R_{1}(\alpha)$ $=\varnothing$. From (1) and (3) we have that (4) $R_{1}(\alpha)-\operatorname{dom} R_{1}(\alpha) \supset[\alpha \beta]$. We also have that $(5) T_{1}(\alpha)-\operatorname{dom} R_{1}(\alpha)=T_{1}(\alpha)-\operatorname{dom}\{\alpha, \beta\}=\left\{x: x \in T_{1}(\alpha), x^{2} \geqq \alpha^{2}, x^{3} \geqq \beta^{3}\right\}$. Define $\mu=\left(e-\alpha^{2}-\beta^{3}, \alpha^{2}, \beta^{3}\right)$.

III.b.1. $\mu \notin A$. By I.4 we have that (6) $\left\{x: x^{S_{1}} \geqq \mu^{S_{1}}\right\} \cap A=\varnothing$. By (2) and II.1 the $R_{1}(\alpha)$-core is the solution of $R_{1}(\alpha)$. By (4), (5) and (6) we have that $R_{1}(\alpha)$ - dom $R_{1}(\alpha)$ solves $A$.

III.b.2. $\mu \in A$. $\mu \in D^{1}-\AA^{1}$ therefore $Q_{1}(\mu)$ is solvable. We remark that (7) $Q_{1}(\mu) \cap A^{1} \subset\left\{x: x^{2}=\alpha^{2}\right\}$. We distinguish several subcases of II.b.2.

III.b.2.1. There is a solution $V_{1}$ of $Q_{1}(\mu)$ such that $V_{1} \cap A^{1}=\emptyset$. We have that (8) $\operatorname{dom} V_{1} \cap R_{1}(\alpha)=\varnothing$. From (5) and (8) it follows that

is a solution of $G$.

$$
V_{1} \cup\left(R_{1}(\alpha)-\operatorname{dom} R_{1}(\alpha)\right)
$$

III.b.2.2. There is a solution $V_{2}$ of $Q_{1}(\mu)$ such that $V_{2} \cap A^{1}=\{\mu\}$. In this case: $R_{1}(\alpha)-\operatorname{dom} V_{2}=Q_{3}(\alpha) \cup Q_{2}(\beta) . \alpha \in D^{3}-\AA^{3}$ and $\beta \in D^{2}-A^{2}$ so $Q_{3}(\alpha)$ and $Q_{2}(\beta)$ are solvable and if $U$ solves $Q_{3}(\alpha)$ and $W$ solves $Q_{2}(\beta)$ then, by I.13, $\alpha \in U$ and $\beta \in W$. Since $Q_{3}(\alpha) \subset R_{2}(\beta)$ and $Q_{2}(\beta) \subset R_{3}(\alpha)$ it follows from I.12 that

$$
\operatorname{dom} U \cap W=\operatorname{dom} W \cap U=\varnothing .
$$

From these results and (5) it follows that $U \cup W \cup V_{2}$ is a solution of $G$.

III.b.2.3. There is a solution $V_{3}$ of $Q_{1}(\mu)$ such that $V_{3} \cap A^{1}-\{\mu\} \neq \varnothing$. Let $x^{3}$ receive its maximum in $V_{3} \cap A^{1}$ at the point $\zeta \cdot \zeta^{2}=\mu^{2}$ and $\zeta^{3}>\mu^{3}$. By II.3 $[\mu \alpha] \cup V_{3}=V_{3}^{\prime}$ solves $Q_{1}(\alpha)$. We define $\nu=\left(\alpha^{1}, e-\alpha^{1}-\zeta^{3}, \zeta^{3}\right) . R_{1}(\alpha)-\operatorname{dom} V_{3}^{\prime}$ $=Q_{3}(\alpha) \cup\left\{x: x \in A, x^{S_{2}} \geqq v^{s_{2}}\right\}$. Let $U$ solve $Q_{3}(\alpha)$. $\operatorname{dom} U \cap\left(Q_{1}(\alpha) \cup\{x\right.$ : $\left.\left.x \in A, x^{S_{2}} \geqq v^{s_{2}}\right\}\right)=\emptyset$. If $v \notin A$ then $V_{3}^{\prime} \cup U$ solves $G$. If $v \in A$ then $v \in D^{2}-A^{2}$. By I.16 $Q_{2}(v) \cap A^{2}=\emptyset . \quad Q_{1}(\alpha) \cup Q_{3}(\alpha) \subset R_{2}(v)$ therefore by $1.12 \operatorname{dom} Q_{2}(v)$ $\cap\left(Q_{1}(\alpha) \cup Q_{3}(\alpha)\right)=\varnothing$. If $W$ solves $Q_{2}(v)$ we have that $V_{3}^{\prime} \cup U \cup W$ is a solution of $G$.

III.c. $F^{1}$ has $c_{3}$-shape. As in III.a, we have that $R_{1}(\alpha)$ is solvable and if $Q_{1}(\alpha)$ and $R_{1}(\alpha)$ are independent then $G$ is solvable. If $Q_{1}(\alpha)$ and $R_{1}(\alpha)$ are not independent and $V$ solves $Q_{1}(\alpha)$ and $W R_{1}(\alpha)$ then either dom $V \cap W \neq \varnothing$ or dom $W \cap V \neq \varnothing$.

III.c.1. There exist $V_{0}$ and $W_{0}$ such that $\operatorname{dom} V_{0} \cap W_{0} \neq \emptyset . \alpha \in W_{0}$ therefore there must be a $z \neq \alpha$ in $V_{0} \cap A^{1}$. We have that either $z^{3}=\alpha^{3}$ or $z^{2}=\alpha^{2}$. 
III.c.1.1. $z^{3}=\alpha^{3}$. In this case we have that (1) $y \in A^{1}$ implies $y^{3} \leqq z^{3}$. Let $x^{2}$ take its maximum in $V_{0} \cap A^{1}$ at the point $\zeta$. Define $v=\left(\alpha^{1}, \zeta^{2}, e-\alpha^{1}-\zeta^{2}\right)$. From (1) it follows that (2) $R_{1}(\alpha)-\operatorname{dom} V_{0}=Q_{2}(\alpha) \cup\left\{x: x \in A, x^{S_{3}} \geqq v^{S_{3}}\right\}$. By II.5 there is a solution $U$ of $Q_{2}(\alpha)$ such that $U \cap F^{1}=\{\alpha\}$; it follows that (3) $\operatorname{dom} U \cap Q_{1}(\alpha)=\varnothing$. If $v \notin A$ then $U \cup V_{0}$ is a solution of $G$. If $v \in A$ then $v \in D^{3}$ $-A^{3}$, so $Q_{3}(v) \cap A^{3}=\varnothing$. It follows that (4) $\operatorname{dom} Q_{3}(v) \cap\left(Q_{2}(\alpha) \cup Q_{1}(\alpha)\right)=\varnothing$. We also have that (5) $\operatorname{dom} U \cap Q_{3}(v)=\varnothing$. Now if $U_{1}$ is a solution of $Q_{3}(v)$ then, combining (2), (3), (4) and (5), we have that $V_{0} \cup U \cup U_{1}$ is a solution of $G$.

III.c.1.2. $z^{2}=\alpha^{2}$. We now have that $y \in A^{1}$ implies $y^{2} \leqq \alpha^{2}$. We show that we may suppose: $(*)$ there is no $u \in A^{3}$ such that $u^{2}=\alpha^{2}$ and $u^{1}>\alpha^{1}$. If (*) fails then $F^{2}$ has $b$-shape and $\left\{A^{1}, A^{3}\right\}$ satisfies condition $M$, so by III.b $G$ is solvable. We also notice that: $(* *)$ if $\vartheta \in A, \vartheta^{2}=\alpha^{2}, \vartheta^{3} \leqq \beta^{3}$ and $J$ solves $Q_{1}(\vartheta)$ then $J \cup[\alpha \vartheta]$ solves $Q_{1}(\alpha)$. Now if $(*)$ holds and $U$ is a solution of $Q_{3}(\alpha)$ then $\operatorname{dom} U$ $\cap\left(Q_{1}(\alpha) \cup Q_{2}(\alpha)\right)=\varnothing$. Let $V$ solve $Q_{1}(\alpha)$. We denote by $\mu(V)$ the point where $x^{3}$ takes its maximum in $A^{1} \cap V$. The point where $x^{3}$ takes its maximum in $A^{1} \cap$ $\left\{x: x^{1}=\alpha^{1}\right\}$ is denoted by $\zeta$. $U$ denotes a fixed solution of $Q_{3}(\alpha)$. We remark that $\alpha \in U$.

III.c.1.2.1. There is a solution $V_{1}$ of $Q_{1}(\alpha)$ such that $\beta^{3}<\mu^{3}\left(V_{1}\right)$. Define $v$ $=\left(\alpha^{1}, e-\alpha^{1}-\mu^{3}\left(V_{1}\right), \mu^{3}\left(V_{1}\right)\right)$. We have that $R_{1}(\alpha)-\operatorname{dom} V_{1}=Q_{3}(\alpha) \cup\{x$ : $\left.x \in A, x^{S_{2}} \geqq v^{S_{2}}\right\}$. If $v \notin A$ then $U \cup V_{1}$ is a solution of $G$. If $v \in A$ then $v \in D^{2}$ $-A^{2}$. Let $U_{1}$ solve $Q_{2}(v)$. $\operatorname{dom} U_{1} \cap\left(Q_{1}(\alpha) \cup Q_{3}(\alpha)\right)=\varnothing$, so $U \cup U_{1} \cup V_{1}$ is a solution of $G$.

III.c.1.2.2. Every solution $V$ of $Q_{1}(\alpha)$ satisfies $\mu^{3}(V) \leqq \beta^{3}$ and there is a solution $V_{1}$ of $Q_{1}(\alpha)$ such that $\mu^{3}\left(V_{1}\right)=\beta^{3} . \beta \in a^{2} \cap D^{2}$ therefore $Q_{2}(\beta)$ is solvable. If $U_{1}$ solves $Q_{2}(\beta)$ then $U_{1} \cap A^{2}=\{\beta\}$ and $\operatorname{dom} U_{1} \cap\left(Q_{1}\left(\mu\left(V_{1}\right)\right) \cup Q_{3}(\alpha)\right)$ $=\varnothing$. Let $U_{2}$ be a solution of $Q_{1}\left(\mu\left(V_{1}\right)\right)$. From $(* *)$ it follows that $U_{2} \cap A^{1}=$ $\left\{\mu\left(V_{1}\right)\right\}$. So we have that $U \cup U_{1} \cup U_{2}$ is a solution of $G$.

III.c.1.2.3. If $V$ solves $Q_{1}(\alpha)$ then $\mu^{3}(V)<\beta^{3}$.

III.c.1.2.3.1. $\zeta^{3} \geqq \beta^{3}$. Define $v=\left(e-\alpha^{2}-\beta^{3}, \alpha^{2}, \beta^{3}\right)$. By I.17 $Q_{1}(\alpha) \cap \operatorname{dom}$ $R_{1}(\alpha)=\operatorname{dom}[\alpha \beta] \cap Q_{1}(\alpha)$, so $\operatorname{dom} R_{1}(\alpha) \cap\left\{x: x \in A, x^{s_{1}} \geqq v^{s_{1}}\right\}=\varnothing$. If $v \notin A$ and $U_{1}$ solves $Q_{2}(\beta)$ then $U \cup[\alpha \beta] \cup U_{1}$ solves $G$. If $v \in A$ let $U_{2}$ be a solution of $Q_{1}(v)$. By $(* *)$ and the definition of $U_{2} U_{2} \cap A^{1}=\varnothing$. So in this case $U \cup U_{1}$ $\cup U_{2} \cup[\alpha \beta]$ solves $G$.

III.c.1.2.3.2. $\zeta^{3}<\beta^{3}$. Define $v=\left(e-\alpha^{2}-\zeta^{3}, \alpha^{2}, \zeta^{3}\right)$. By II.5 there is a solution $U_{1}$ of $Q_{2}(\zeta)$ such that $U_{1} \cap F^{1}=\{\zeta\}$. If $v \notin A$ then $U \cup U_{1} \cup[\alpha \zeta]$ is a solution of $G$. If $v \in A-A^{1}$ and $U_{2}$ solves $Q_{1}(v)$ then $U \cup U_{1} \cup U_{2} \cup[\alpha \zeta]$ is a solution of $G$. If $v \in A^{1}$ then $U_{1} \cup U_{2} \cup U$ is a solution of $G$.

III.c.2. If $V$ solves $Q_{1}(\alpha)$ and $W R_{1}(\alpha)$ then $\operatorname{dom} W \cap V \neq \emptyset$ and $\operatorname{dom} V \cap W$ $=\varnothing$. By I.17 we have that $\operatorname{dom}\left(R_{1}(\alpha)-[\beta \alpha)\right) \cap Q_{1}(\alpha)=\emptyset$. By I.19 $\stackrel{\circ}{R}_{1}^{3}(\alpha)=\AA^{3}$ $\cap R_{1}(\alpha) .[\beta \alpha) \subset \AA^{3}$ therefore $[\beta \alpha) \subset \stackrel{R}{1}_{1}^{3}(\alpha)$. So we conclude that

$$
\operatorname{dom}\left(R_{1}(\alpha)-\operatorname{dom} R_{1}(\alpha)\right) \cap Q_{1}(\alpha)=\varnothing .
$$


Since $F^{1}$ has $c_{3}$-shape we have also that $\AA^{2} \cap R_{1}(\alpha)=\varnothing$. If $\left\{A^{1}, A^{3}\right\}$ does not intersect maximally then, by I.20, $\left\{R_{1}^{1}(\alpha), R_{1}^{3}(\alpha)\right\}$ does not intersect maximally and we have that $m\left(R_{1}(\alpha)\right)=0$. By II.2 the $R_{1}(\alpha)$-core is the solution of $R_{1}(\alpha)$ which, by (1), contradicts III.c.2. So $\left\{A^{1}, A^{3}\right\}$ intersects maximally. The $x^{2}$ coordinate of $F^{2}$ will be denoted by $d$.

III.c.2.1. $d<\alpha^{2}$. We denote the ends of $F^{2}$ by $\gamma$ and $\delta$ such that $\delta^{1} \geqq \gamma^{1}$. We remark that $\delta \in D^{2}-A^{2}$. So $Q_{2}(\delta)$ is solvable and if $U$ solves it then $\operatorname{dom} U \supset T_{2}(\delta)$ $-U$ and $\operatorname{dom} U \cap R_{2}(\delta)=\varnothing$. Denote $v=\left(\alpha^{1}, d, e-d-\alpha^{1}\right)$ and $P=\{x$ : $\left.x \in A, x^{S_{3}} \geqq v^{S_{3}}\right\}$. We remark that $\stackrel{\circ}{P}^{i} \cap \stackrel{\circ}{ }^{j}=\varnothing$ for all $i \neq j$ and that $(P-\operatorname{dom} P)$ $\cap((\alpha \beta] \cup[\gamma \delta))=\varnothing . \operatorname{So}\left({ }^{3}\right) P-\operatorname{dom} P$ solves $P$ and

$$
\operatorname{dom}(P-\operatorname{dom} P) \cap\left(Q_{2}(\delta) \cup Q_{1}(\alpha)\right)=\varnothing .
$$

Summing we have that $U_{1}=U \cup(P-\operatorname{dom} P)$ solves $R_{1}(\alpha)$ and that $\operatorname{dom} U_{1} \cap$ $Q_{1}(\alpha)=\varnothing$. Since this result contradicts III.c. $2 d<\alpha^{2}$ is impossible.

III.c.2.2. $d=\alpha^{2}$. If $F^{2}$ has $a$-shape or $b$-shape then $\left\{A^{1}, A^{3}\right\}$ satisfies condition $M$ and by III.a or III.b $G$ is solvable. It remains only to complete the proof when $F^{2}$ has $c_{3}$ or $c_{1}$-shape.

III.c.2.2.1. $\gamma^{1}<\alpha^{1}$. In this case $F^{2}$ has $c_{3}$-shape. Let $\eta$ satisfy $\eta^{2}=\alpha^{2}$ and $\alpha^{1}>\eta^{1}>\max .\left(\gamma^{1}, e-\alpha^{2}-\beta^{3}\right)$ and $U_{1}$ be a solution of $Q_{1}(\eta) . U_{2}=[\alpha \eta] \cup U_{1}$ solves $Q_{1}(\alpha)$. Let $\zeta \in(\alpha \beta] \cap A^{1}, U_{3}$ be a solution of $Q_{2}(\zeta)$ and $U_{4}$ a solution of $Q_{3}(\alpha)$. $U_{5}=U_{3} \cup U_{4} \cup[\alpha \zeta]$ is a solution of $R_{1}(\alpha)$. But dom $U_{2} \cap U_{5} \neq \varnothing$ contradicting III.c.2, so III.c.2.2.1 is impossible.

III.c.2.2.2. $\gamma^{1}=\alpha^{1}$. In this case $F^{2}$ has $c_{1}$-shape. By II.5 there exist solutions $U_{1}$ of $Q_{3}(\alpha)$ and $U_{2}$ of $Q_{2}(\alpha)$ such that $U_{1} \cap F^{2}=\{\alpha\}=U_{2} \cap F^{1} . U=U_{1} \cup U_{2}$ is a solution of $R_{1}(\alpha)$ but $\left({ }^{3}\right)$ dom $U \cap Q_{1}(\alpha)=\varnothing$ contradicting III.c.2.

III.c.2.2.3. $\gamma^{1}>\alpha^{1}$. In this case $F^{2}$ has $c_{1}$-shape and $\gamma \in a^{1} \cap a^{3}-A^{2}$. Let $U$ solve $Q_{2}(\gamma) . U_{1}=U \cup\left(Q_{3}(\alpha)-\operatorname{dom} Q_{3}(\alpha)\right)$ solves $R_{1}(\alpha)$ but $\operatorname{dom} U_{1} \cap Q_{1}(\alpha)=\varnothing$ which is impossible.

III.c.2.3. $d>\alpha^{2}$. In this case we show that $\AA^{1} \cap \AA^{3} \cap R_{1}(\alpha)=\varnothing$. It follows that $\left\{R_{3}^{1}(\alpha), R_{3}^{3}(\alpha)\right\}$ does not intersect maximally which is impossible as we have already seen. Suppose that $\AA^{1} \cap \AA^{3} \cap R_{1}(\alpha) \neq \emptyset$. Let $x \in \mathscr{A}^{1} \cap \cap A^{3} \cap R_{1}(\alpha)$. $x^{1} \geqq \alpha^{1}$. Since $\left({ }^{4}\right) \alpha \in a^{3} x^{2}<\alpha^{2}$. Let $z \in a^{1} \cap a^{3} \cap F^{2}$ and $y$ be an interior point of $A^{\circ} \cap A^{\circ}$. There is a $u \in[y z] \cap A^{1} \cap A^{\circ}$ such that $u^{2}>\alpha^{2}$. So there is a $w \in[u x]$ for which $w^{2}=\alpha^{2} \cdot w \in \AA^{1} \cap \AA^{3}$. If $w^{1} \geqq \alpha^{1}$ then $\alpha \in \AA^{3}$ and if $w^{3} \geqq \alpha^{3}$ then $\alpha \in \AA^{1}$. Since both cases are impossible we must have $\emptyset=\AA^{1} \cap \AA^{3} \cap R_{1}(\alpha)$.

IV. Third part: case II.9.

IV.1. $m(G) \leqq 2$. W.L.G. $\left\{A^{2}, A^{3}\right\}$ does not intersect maximally.

IV.1.1. $A^{2} \cap \AA^{3}=\varnothing$. We shall show that $m(G)=0$. If $m(G)>0$ then, W.L.G., $\left\{A^{1}, A^{3}\right\}$ intersects maximally. Let $z \in a^{1} \cap a^{3} \cap F^{2}$ and $y$ be an interior point of

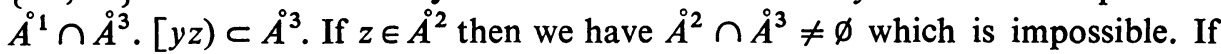

(3) See I.1\%

(4) If $x^{2} \geqq x^{2}$ then $\alpha S_{3} \geqq \alpha S_{3}$ and $x \in \AA^{3}$ imply $\alpha \in \AA^{3}$ which is untrue. 
$z \notin \AA^{2}$ then $\left\{A^{1}, A^{3}\right\}$ satisfies condition $M$, which is again impossible. Therefore $\left\{A^{1}, A^{3}\right\}$ does not intersect maximally.

IV.1.2. $\AA^{3} \supset A^{2}$. If $\left\{A^{1}, A^{3}\right\}$ intersects maximally then there is a $z \in a^{1} \cap a^{3}$ $\cap F^{2} . a^{3} \cap A^{2}=\varnothing$; therefore $z \notin \AA^{2}$. But since we have $a^{1} \cap a^{3} \cap F^{2} \subset A^{2}$, $\left\{A^{1}, A^{3}\right\}$ cannot intersect maximally.

IV.1.2.1. $\AA^{1} \cap \AA^{3}=\emptyset . \AA^{2} \cap \AA^{1} \subset \AA^{1} \cap A^{2} \subset \AA^{1} \cap \AA^{3}=\emptyset$, so $m(G)=0$.

IV.1.2.2. $\AA^{3} \supset A^{1}$. We denote $C=A-\operatorname{dom} A$. If $x \in A-C$ then there is a $y \in A$ such that $y \succ s_{3} x$. So there is a $z \in a^{3}$ such that $z \succ x . a^{3} \cap\left(\AA^{1} \cup \AA^{2}\right)=\varnothing$ therefore $z \in C$. By $2.6 C$ is a solution of $G$.

IV.1.2.3. $\AA^{1} \supset A^{3}$. We have $\AA^{1} \supset A^{3} \supset \AA^{3} \supset A^{2}$, so $m(G)=0$.

IV.1.3. $\AA^{2} \supset A^{3}$. The proof in this case parallels that in IV.1.2.

IV.2. $m(G)=3$. We denote $F^{k}=\left[\alpha_{k} \beta_{k}\right]$ and $D=\bigcap_{h=1}^{3} A^{h}$.

LEMMA IV.2.1. Under the assumptions of IV.2 we can find $i$ and $k$ such that $S_{k}=\{i, j\}$ and $:$

(1) $\alpha_{k}^{i} \geqq \beta_{k}^{i}$

(2) $\alpha_{k} \in a^{i} \cap a^{j}$

(3.a) $x^{j}$ takes its maximum in $\left\{x: x^{i}=\alpha_{k}^{i}\right\} \cap D$ at a point $\vartheta \in a^{k}$ such that every $y \in A^{k}$ that satisfies $y^{j}=\vartheta^{j}$ and $y^{i}>\vartheta^{i}$ is in $A^{i}$, or

(3.b) $x^{i}$ takes its maximum in $\left\{x: x^{j}=\alpha_{k}^{j}\right\} \cap D$ at a point $\rho \in a^{k} \cap \AA^{j}$ and $F^{k}$ has $c_{j}$-shape.

Proof. $F^{1}=\left[\alpha_{1} \beta_{1}\right]$.W.L.G. $\alpha_{1}^{2} \geqq \beta_{1}^{2}$. We also suppose that $\alpha \in a^{2} \cap a^{3}$. If $\alpha \notin a^{2} \cap a^{3}$ then $\beta \in a^{2} \cap a^{3}$ and the proof is not altered much. We now consider $F^{3}$.

IV.2.1.1. Every $y \in F^{3}$ satisfies $y^{2}<\alpha_{1}^{2}$. Let $\vartheta$ be the point where $x^{3}$ takes its maximum in $\left\{x: x^{2}=\alpha_{1}^{2}\right\} \cap D . \vartheta \neq \alpha_{1}$. We show that $\vartheta \in \AA^{2}$. If $F^{3}=\left[\alpha_{3} \beta_{3}\right]$ and $\alpha_{3}^{1} \geqq \beta_{3}^{1}$ then $\alpha_{3}^{3}>\vartheta^{3}$ since $\vartheta \notin F^{3}$. If $\alpha_{3}^{1}>\vartheta^{1}$ then $\alpha_{3} \succ_{s_{2}} \vartheta$, and if $\vartheta^{1} \geqq \alpha_{3}^{1}$ then there is a $u \in\left[\alpha_{1} \alpha_{3}\right]$ such that $u \zeta_{S_{2}} \vartheta$. Now if $\vartheta \in a^{3}$ then $F^{2}$ has $c_{1}$-shape and $\vartheta \in a^{1}$ and if $\vartheta \in \AA^{3}$ then it follows from I.3 that $\vartheta \in a^{1}$. So in this case we can choose $k=1$ and $i=2$.

IV.2.1.2. There is a $y \in F^{3}$ such that $y^{2}=\alpha_{1}^{2}$. Let $\vartheta=y$. If $F^{3}$ has $a$-shape then $\vartheta \in a^{1}$ and there is no $u \in A^{1}$ that satisfies $u^{3}=\vartheta^{3}$ and $u^{2}>\vartheta^{2}$. So we can choose $k=1$ and $i=2$. If $F^{3}$ has not $a$-shape then $\beta_{3}^{2}>\alpha_{3}^{2}$. If $F^{3}$ has $b$-shape and $y=\beta_{3}$ then there is no $u \in A^{1}$ such that $u^{3}=\vartheta^{3}$, and $u^{2}>\vartheta^{2}$ and we can choose $k=1$ and $i=2$. If $y \neq \beta_{3}$ then we have that $\beta_{3} \in a^{2} \cap a^{1}$ and every $x \in F^{1}$ satisfies $x^{2}<\beta_{3}^{2}$. By IV.2.1.1. we may take $k=3$ and $i=2$. If $F^{3}$ has $c_{2}$-shape then $\vartheta \in a$ and if $u \in A, u^{3}=\vartheta^{3}$ and $u^{2}>\vartheta^{2}$ then $u \in A^{2}$. So we can take $k=1$ and $i=2$. If $F^{3}$ has $c_{1}$-shape and $y=\beta_{3}$ we choose $k=1$ and $i=2$. If $y \neq \beta_{3}$ then we have that $\beta_{3} \in a^{2} \cap a^{1}$ and every $x \in F^{1}$ satisfies $x^{2}<\beta_{3}^{2}$. By IV.2.1.1. we can choose $k=3$ and $i=2$.

IV.2.1.3. Every $y \in F^{3}$ satisfies $y^{2}>\alpha_{1}^{2}$. If $F^{3}$ has $a, b$ or $c_{1}$-shape then we have that $\beta_{3}^{2} \geqq \alpha_{3}^{2}, \beta_{3} \in a^{2} \cap a^{1}$ and every $x \in F^{1}$ satisfies $x^{2}<\beta_{3}^{2}$. By IV.2.1.1, we can choose $k=3$ and $i=2$. Now suppose that $F^{3}$ has $c_{2}$-shape. Let $x^{1}$ take its maxi- 
mum in $D \cap\left\{x: x^{2}=\alpha_{3}^{2}\right\}$ at the point $\rho . \rho \neq \alpha_{3}$. We shall show that $\rho \in \AA^{1} \cap \AA^{2}$. $\rho^{2}=\alpha_{3}^{2}<\beta_{3}^{2}$. $\rho^{1}>\alpha_{3}^{1}$ therefore $\rho^{3}<\alpha_{3}^{3}=\beta_{3}^{3}$. So $\beta_{3} \succ_{s_{1}} \rho$. We have also that $\alpha_{1}^{1}>\rho^{1}$ therefore there is a $u \in\left[\alpha_{1} \alpha_{3}\right]$ such that $u \succ_{s_{2}} \rho$. It follows from I.3 that $\rho \in a^{3}$. Summing we have: $\alpha_{3}^{1} \geqq \beta_{3}^{1}, \alpha_{3} \in a^{2} \cap a^{1}$ and $x^{1}$ takes its maximum in $D \cap\left\{x: x^{2}=\alpha_{3}^{2}\right\}$ at a point $\rho \in a^{3} \cap \AA^{2}$. So we can take $k=3$ and $i=1$.

We now prove that $G$ is solvable in case IV.2. W.L.G. the results of IV.2.1 hold for $k=1$ and $i=2$.

IV.2.2. (3.a) holds in IV.2.1. We remark $\left(^{5}\right)$ that if $z \in A^{1} \cap Q_{1}(\vartheta)$ then $z^{3}=\vartheta^{3}$. If there is a $z \in A^{1}$ such that $z^{3}=\vartheta^{3}$ and $z^{2}>\vartheta^{2}$ then $F^{3}$ has $c_{2}$-shape. By Lemma II.5 there is a solution $V$ of $Q_{1}(\vartheta)$ such that $V \cap F^{3}=\{\vartheta\}$. So we can always find a solution $V_{1}$ of $Q_{1}(\vartheta)$ such that $V_{1} \cap A^{1}=\{\vartheta\}$. Similar reasoning shows that there is always a solution $V_{2}$ of $Q_{3}\left(\alpha_{1}\right)$ such that $V_{2} \cap A^{3}=\left\{\alpha_{1}\right\}$.

IV.2.2.1. $\vartheta^{3}>\beta_{1}^{3}$. We define $v=\left(\alpha_{1}^{1}, e-\alpha_{1}^{1}-\vartheta^{3}, \vartheta^{3}\right)$. Suppose $v \in A$. We have that $Q_{2}(v) \cap A^{2}=\varnothing$. So if $U$ is a solution of $Q_{2}(v)$ then $V_{1} \cup V_{2} \cup U \cup\left[\vartheta \alpha_{1}\right]$ solves $G$. If $v \notin A$ then $V_{1} \cup V_{2} \cup\left[\vartheta \alpha_{1}\right]$ solves $G$.

IV.2.2.2. $\vartheta^{3}=\beta_{1}^{3}$. If $U$ is a solution of $Q_{2}\left(\beta_{1}\right)$ then $V_{1} \cup V_{2} \cup U$ is a solution of $G$.

IV.2.2.3. $\vartheta^{3}<\beta_{1}^{3}$. Let $x^{3}$ take its maximum in $A^{1} \cap\left\{x: x^{1}=\alpha_{1}^{1}\right\}$ at $\zeta$. We define $\mu=\left(e-\alpha_{1}^{2}-\beta_{1}^{3}, \alpha_{1}^{2}, \beta_{1}^{3}\right)$. Suppose $\zeta^{3} \geqq \beta_{1}^{3}$ and $\mu \in A$. In this case if $W$ is a solution of $Q_{2}\left(\beta_{1}\right)$ and $W_{1}$ is a solution of $Q_{1}(\mu)$ then $V_{2} \cup W \cup W_{1} \cup F^{1}$ solves $G$. If $\mu \notin A$ then $V_{2} \cup W \cup F^{1}$ is a solution of $G$. If $\zeta^{3}<\beta_{1}^{3}$ then $F^{1}$ has $c_{3}$-shape. We define $\eta=\left(e-\zeta^{3}-\alpha_{1}^{2}, \alpha_{1}^{2}, \zeta^{3}\right)$. By II.5 there is a solution $U$ of $Q_{2}(\zeta)$ such that $\{\zeta\}=U \cap F^{1}$. If $\eta \notin A$ then $U \cup V_{2} \cup\left[\zeta \alpha_{1}\right]$ solves $G$. If $\eta \in A-A^{1}$ and $U_{1}$ solves $Q_{1}(\eta)$ then $V_{2} \cup U \cup U_{1} \cup\left[\zeta \alpha_{1}\right]$ is a solution for $G$. If $\eta \in A^{1}$ then $\eta=\vartheta$ and $V_{1}$ $\cup V_{2} \cup U$ is a solution of $G$.

IV.2.3.(3.b) holds in IV.2.1. So $F^{1}$ has $c_{3}$-shape. By II.5 there is a solution $V$ of $Q_{2}\left(\alpha_{1}\right)$ such that $V \cap F^{1}=\left\{\alpha_{1}\right\}$. Next we show that there is a solution $V_{1}$ of $Q_{1}(\rho)$ such that $V_{1} \cap A^{1}=\{\rho\}$. If $Q_{1}(\rho) \cap A^{1}=\{\rho\}$ this follows from the fact that $\rho$ belongs to every solution of $Q_{1}(\rho)$. If there is $x \in Q_{1}(\rho) \cap A^{1}, x \neq \rho$, then $x \in a^{1}$ and $\left(^{6}\right) x^{2}=\rho^{2}$. Using $I .10$ and observing that $\rho \in \AA^{3}$ we see that $F^{2}$ has $C_{3}$-shape and $\rho \in F^{2}$. II.5 yields a desired $V_{1}$. Now define $v=\left(\alpha_{1}^{1}, \rho^{2}, e-\alpha^{1}-\rho^{2}\right)$. Observe that $Q_{3}(v) \cap A^{3}=\varnothing$. If $v \in A$ and $V_{2}$ is a solution of $Q_{3}(v)$ then $V \cup V_{1}$ $\cup V_{2} \cup\left[\rho \alpha_{1}\right]$ is a solution of $G$. If $v \notin A$ then $V \cup V_{1} \cup\left[\rho \alpha_{1}\right]$ solves $G$.

\section{REFERENCES}

1. R. J. Aumann and B. Peleg, Von Neumann Morgenstern solutions to cooperative games without side payments, Bull. Amer. Math. Soc. 66 (1960), 173-179.

2. R. J. Aumann, The core of cooperative game without side payments, Trans. Amer. Math. Soc. 98 (1961), 539-552.

3. D. B. Gillies, Solutions to general non-zero-sum games, Contributions to theory of games. IV, pp. 47-85, Princeton Univ. Press, Princeton, N. J., 1959.

THE HeBrew UNIVERSITY, JERUSALEM, ISRAEL

(5) Suppose there is $z \in A^{1} \cap Q_{1}(\vartheta)$ with $z 3>\vartheta^{3} . \alpha_{1} \in \stackrel{\circ}{A}^{1}$ so there is $u \in A^{1}$ with $u^{2}>\alpha_{2}^{1}=\vartheta^{2}$. For small $t>0 y=t u+(1-t) z$ satisfy $y^{S_{1}}>\vartheta^{S_{1}}$ and $y \in A^{1}$ which is impossible since $\vartheta \in a^{1}$.

(6) By an argument similar to that in footnote (5). 